\title{
3-D Velocity Estimation for Two Planes in vivo
}

Holbek, Simon; Pihl, Michael Johannes; Ewertsen, Caroline; Bachmann, Michael Bachmann; Jensen, Jørgen Arendt

\section{Published in:}

Proceedings of IEEE International Ultrasonics Symposium

Link to article, DOI:

10.1109/ULTSYM.2014.0423

Publication date:

2014

Document Version

Early version, also known as pre-print

Link back to DTU Orbit

Citation (APA):

Holbek, S., Pihl, M. J., Ewertsen, C., Bachmann, M. B., \& Jensen, J. A. (2014). 3-D Velocity Estimation for Two Planes in vivo. In Proceedings of IEEE International Ultrasonics Symposium (pp. 1706-1709). IEEE. https://doi.org/10.1109/ULTSYM.2014.0423

\section{General rights}

Copyright and moral rights for the publications made accessible in the public portal are retained by the authors and/or other copyright owners and it is a condition of accessing publications that users recognise and abide by the legal requirements associated with these rights.

- Users may download and print one copy of any publication from the public portal for the purpose of private study or research.

- You may not further distribute the material or use it for any profit-making activity or commercial gain

- You may freely distribute the URL identifying the publication in the public portal

If you believe that this document breaches copyright please contact us providing details, and we will remove access to the work immediately and investigate your claim 
Paper presented at the IEEE International Ultrasonics Symposium, Chicago, Il., USA, 2014:

\section{3-D Velocity Estimation for Two Planes in vivo}

Simon Holbek ${ }^{1}$, Michael Johannes Pihl ${ }^{1}$, Caroline Ewertsen ${ }^{2}$, Michael Bachmann Nielsen ${ }^{2}$ and Jorgen Arendt Jensen ${ }^{1}$

${ }^{1}$ Center for Fast Ultrasound Imaging,

Biomedical Engineering group, Department of Electrical Engineering, Bldg. 349, Technical University of Denmark, DK-2800 Kgs. Lyngby, Denmark

${ }^{2}$ Department of Radiology, Rigshospitalet, Copenhagen University Hospital, Denmark 


\title{
3-D Velocity Estimation for Two Planes in vivo
}

\author{
Simon Holbek*, Michael Johannes Pihl*, Caroline Ewertsen ${ }^{\dagger}$, Michael Bachmann Nielsen ${ }^{\dagger}$ and Jørgen Arendt Jensen* \\ * Center for Fast Ultrasound Imaging, Dept. of Elec. Eng., Bldg. 349, Technical University of Denmark, \\ 2800 Kgs. Lyngby, Denmark \\ $\dagger$ Department of Radiology, Copenhagen University Hospital, \\ 2100 Copenhagen, Denmark
}

\begin{abstract}
D velocity vectors can provide additional flow information applicable for diagnosing cardiovascular diseases e.g. by estimating the out-of-plane velocity component. A 3-D version of the Transverse Oscillation (TO) method has previously been used to obtain this information in a carotid flow phantom with constant flow. This paper presents the first in vivo measurements of the 3-D velocity vector, which were obtained over 3 cardiac cycles in the common carotid artery of a 32-year-old healthy male volunteer. Data were acquired using a Vermon $3.5 \mathrm{MHz}$ $32 \times 32$ element 2-D phased array transducer and stored on the experimental scanner SARUS. The full 3-D velocity profile can be created and examined at peak-systole and end-diastole without ECG gating in two planes. Maximum out-of-plane velocities for the three peak-systoles and end-diastoles were $68.5 \pm 5.1 \mathrm{~cm} / \mathrm{s}$ and $26.3 \pm 3.3 \mathrm{~cm} / \mathrm{s}$, respectively. In the longitudinal plane, average maximum peak velocity in flow direction was $65.2 \pm 14.0 \mathrm{~cm} / \mathrm{s}$ at peak-systole and $33.6 \pm 4.3 \mathrm{~cm} / \mathrm{s}$ at end-diastole. A commercial BK Medical ProFocus UltraView scanner using a spectral estimator gave $79.3 \mathrm{~cm} / \mathrm{s}$ and $14.6 \mathrm{~cm} / \mathrm{s}$ for the same volunteer. This demonstrates that real-time 3-D vector velocity imaging without ECG gating yields quantitative in vivo estimations on flow direction and magnitude.
\end{abstract}

\section{INTRODUCTION}

Ultrasound (US) is a commonly used approach for estimating blood velocities. The information about the direction and magnitude of the blood flow is of great importance in the clinic for diagnosing various cardiovascular diseases [1] [2]. The possibility of estimating the full 3-D velocity vector in any given plane would provide valuable additional information for the medical doctors. Currently 3-D vector velocity can be acquired with MRI, but this image modality can only show a flow cycle that is averaged from 10-15 minutes of data acquisition [3][4]. Therefore the flow in real-time is not shown. Furthermore, MRI also has the disadvantage compared to ultrasound that the cost is much higher and that it can be more uncomfortable to the patients.

The 3-D Transverse Oscillation (TO) method has previously shown good results for out-of-plane flow rate estimation in a phantom with constant flow, as well as in in vivo Mmode measurements of the 3-D velocity vector at the center of a carotid artery [5]. These values are currently estimated clinically from spectral Doppler velocities. A correct velocity value is obtained when: The vessel has circular symmetry, the US beam penetrates the middle of the vessel, and when the angle correction is done correctly [6]. By implementing the 3-D TO technique to obtain the full 3-D velocity vector in vivo for the out-of-plane components, the accuracy of the measured flow rates and peak velocities in the vessel could be improved, as this approach is unaffected by vessel geometry.
More flexibility in probe placement is also obtained as results are angle independent.

This paper shows that the 3-D TO method can be used to estimate the full 3-D velocity vector in vivo for two crossing scan planes in the common carotid artery for a 32-year-old healthy male volunteer.

\section{Methods}

\section{A. Scanner setup}

An experimental ultrasound scanner SARUS [7] with 1024 channels in receive and transmit was used along with a Vermon 3.5 MHz 32x32 element 2-D phased array transducer (Vermon S.A., Tours, France) for data acquisition. Data were sampled from all 1024 channels and stored for offline processing on a Linux cluster. An illustration of the experimental setup is given in previous work [5].

\section{B. Emissions sequence}

At $13 \mathrm{~Hz}, 45$ frames consisting of two crossing planes were acquired with a complex interleaved sequence composed of 768 emissions. The emission sequence for each plane was as follows (see illustration on Fig. 1): First the left half-plane was created by emitting 5 unique flow emissions running from $\mathrm{F}_{1}$ to $\mathrm{F}_{5}$ consecutively. After the emission of $\mathrm{F}_{5}$ the first Bmode $B_{1}$ shot is emitted. This is followed by the emissions $F_{1-5}$ with $B_{2}$ emitted after the last flow line, $F_{5}$. This pattern continues for 32 cycles and the full sequence for the left halfplane can schematically be written as:

$$
\begin{aligned}
& F_{1} \rightarrow F_{2} \rightarrow F_{3} \rightarrow F_{4} \rightarrow F_{5} \rightarrow B_{1} \rightarrow \\
& F_{1} \rightarrow F_{2} \rightarrow F_{3} \rightarrow F_{4} \rightarrow F_{5} \rightarrow B_{2} \rightarrow \\
& F_{1} \rightarrow F_{2} \rightarrow F_{3} \rightarrow F_{4} \rightarrow F_{5} \rightarrow B_{32} \rightarrow
\end{aligned}
$$

The right half-plane is created in a similar way such that the flow emissions $\mathrm{F}_{5-9}$ are followed by the $\mathrm{B}$-mode line $\mathrm{B}_{33}$ etc. where the flow line $F_{5}$ is the only unique emission that is present in both half-planes. The second plane is created immediately after emission $\mathrm{B}_{64}$ and contains additionally 64 unique B-mode lines and 9 unique flow lines since $\mathrm{F}_{5}$ also is present in this plane. The repetition of $\mathrm{F}_{5}$ in all 4 half-planes allows for velocity estimation of continuous data in the center shot. Each plane consisted of $64 \mathrm{~B}$-mode emissions spanning the angles from $-31.5^{\circ}$ to $31.5^{\circ}$ in steps of $1^{\circ}$ and $32 \times 10$ flow emissions covering the angle span from $-12^{\circ}$ to $12^{\circ}$ in steps of 

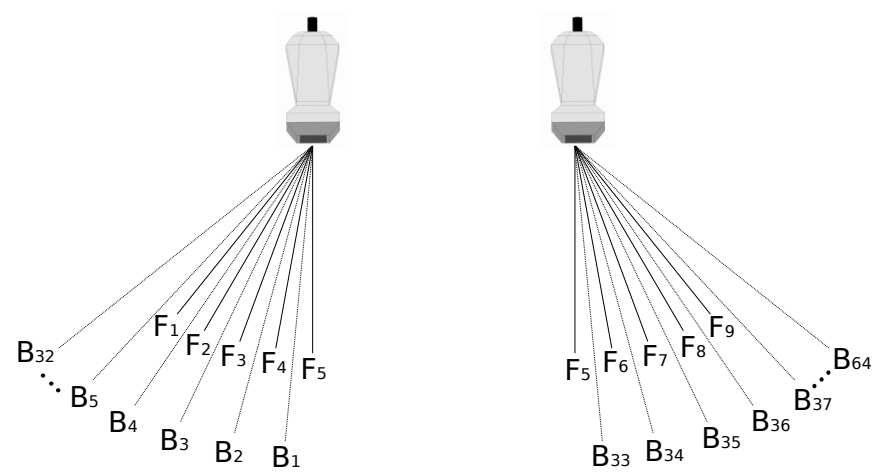

Fig. 1. Left: The emissions sequence for the left half-plane. First the unique flow lines $\mathrm{F}_{1-5}$ are emitted and are followed by the unique B-mode line $B_{1}$. The emission of $B_{1}$ if followed by the emissions $F_{1-5}$ that are followed by $\mathrm{B}_{2}$ and so on. This continues 32 times until $\mathrm{B}_{32}$ is emitted. Right: The emissions sequence for the right half-plane. The emission procedure is the same as for the left half-plane but with new unique flow and B-mode lines. Only $\mathrm{F}_{5}$ is emitted again which ensures that continuous data in the center can be obtained. Note that angles between lines are not representative for the used sequence but serves as an illustration of the principles.

$3^{\circ}$. With a pulse repetition frequency (PRF) of $9.9 \mathrm{kHz}$ and by creating one half-plane at a time, the effective PRF for each flow line was $1.65 \mathrm{kHz}$. With this experimental setup, sequence and sampling depth, a total duration of $3.5 \mathrm{~s}$ of continuous data could be stored.

The reason for constructing such a complex sequence was to increase the number of flow lines used in each plane to cover the region of interest without lowering frame rates to inadequate levels. Also, the effective PRF for each flow line was taken into account in the sequence design, since this, together with the transverse wavelength at a specific depth, determines the maximum velocity that can be estimated without aliasing. The maximum velocities that could be estimated in $2 \mathrm{~cm}$ depth with the auto-correlation method were is our case:

$$
\begin{aligned}
& \max V_{x}=\frac{\lambda_{x}}{4} \cdot P R F=\frac{0.228 \mathrm{~cm}}{4} \cdot 1650 \mathrm{~s}^{-1}=94 \mathrm{~cm} / \mathrm{s} \\
& \max V_{y}=\frac{\lambda_{y}}{4} \cdot P R F=\frac{0.257 \mathrm{~cm}}{4} \cdot 1650 \mathrm{~s}^{-1}=106 \mathrm{~cm} / \mathrm{s}
\end{aligned}
$$

Where $\lambda_{x}$ and $\lambda_{x}$ were the transverse wavelengths. These value were calculated beforehand to ensure that the maximum velocities in the carotid could be estimated.

\section{FDA limits}

Intensity measurements of the complex emission sequence used for the in-vivo measurements were conducted with an acoustic intensity measurement system, AIMS III (Onda, Sunnyvale, California, USA). The derated value for MI was 1.50, and the pulse repetition frequency was scaled to $9.9 \mathrm{kHz}$ to obtain $\mathrm{I}_{\text {spta. } 3}=720 \mathrm{~mW} / \mathrm{cm}^{2}$ in compliance with the FDA limits [8]. Thermal measurements were also conducted for the sequence by measuring the temperature on the surface of the transducer for 30 minutes at continuous emission. The measurements showed a temperature rise of $7.3^{\circ} \mathrm{C}$ in air and a rise in temperature in a tissue mimicking phantom (Danish Phantom Design, Frederikssund, Denmark) of $2.7^{\circ} \mathrm{C}$. Both in compliance with the IEC limits of a maximum temperature rise of $27^{\circ} \mathrm{C}$ in air and $10^{\circ} \mathrm{C}$ for tissue at 30 minutes consecutive emission [9].

\section{Clinical setup}

To ensure a steady flow, the volunteer had been resting for 15 minutes before the measurements were conducted. A spectrogram of the volunteer's common carotid artery was acquired using a BK8670 linear transducer and a BK Medical ProFocus scanner. Since the spectrogram and the vector flow imaging (VFI) data could not be obtained simultaneously, due to use of different scanners and transducers, the VFI data were obtained immediately after the collection of the spectrogram data.

\section{E. Data processing}

The stored data were processed offline, where the raw RF data were match filtered and Hilbert transformed before the IQ data were beamformed with the Beamformation Toolbox 3 [10]. In this part, the three velocity components were decoupled, such that one line was beamformed for the axial velocity estimation and two dedicated lines were beamformed for each of the transverse and elevation velocity estimates. In total 5 unique beamformed lines were used to estimate the 3 -D velocity vector for each flow line. For a more extensive description of the 3-D transverse oscillation method used, see previous work [11]. Echo cancellation of the beamformed data was subsequently done with a low frequency Doppler filter algorithm [12] due to the rapidly moving vessel walls in the peak-systole.

The axial velocity estimates were based on the autocorrelation approach [13] whereas the transverse and elevation estimates relied on the TO method [14][15]. Due to the geometry and the asymmetry of the transducer, two distinct and simulated transverse wavelength were used; $\lambda_{x}=2.28 \mathrm{~mm}$ and $\lambda_{y}=2.57 \mathrm{~mm}$. The estimated velocities were finally rotated according to their steering angle and linearly interpolated. Each velocity estimate was based on a packet size of 32 emissions. The discrimination used to select between flow and B-mode image in the vessel was drawn manually by an experienced radiologist based on one B-mode image.

\section{RESULTS}

Forty-five cross-sectional frames were acquired in which three cardiac cycles could be identified. Figure 2 shows the three velocity components in the transverse out-of-plane for two consecutive frames. The left column shows the velocities at the end-diastole, where the maximum velocities are (30.1, $8.4,1.0) \mathrm{cm} / \mathrm{s}$ in the $\left(v_{x}, v_{y}, v_{z}\right)$ directions respectively. The column to the right are the three velocity components at one of the identified peak-systoles with maximum velocities of (74.3, $28.4,2.5) \mathrm{cm} / \mathrm{s}$.

Figure 3 shows the three velocity components in the longitudinal ZX-plane for two consecutive frames. The left column shows the velocities at the end-diastole, where the maximum velocities are $(35.4,12.5,4.9) \mathrm{cm} / \mathrm{s}$ in the $\left(v_{x}, v_{y}, v_{z}\right) \mathrm{di}-$ rections respectively. The column to the right are the three velocity components at one of the identified peak-systoles with maximum velocities of $(75.0,74.9,20.7) \mathrm{cm} / \mathrm{s}$.

For comparison the similar angle corrected $v_{x}$ velocities estimated with spectral Doppler on a commercial scanner in the middle of the vessel and along the direction of flow were 

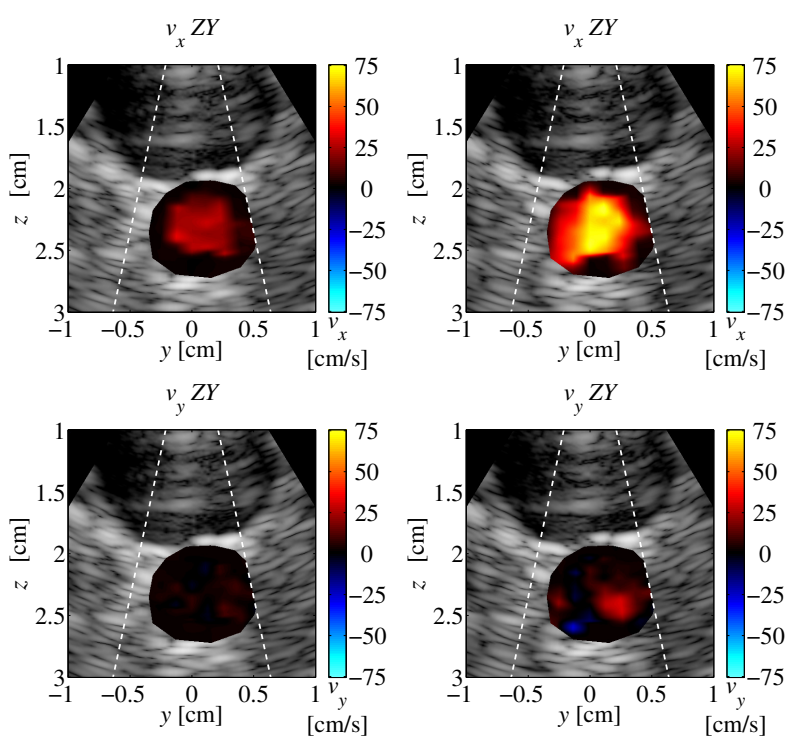

$v_{z} Z Y$
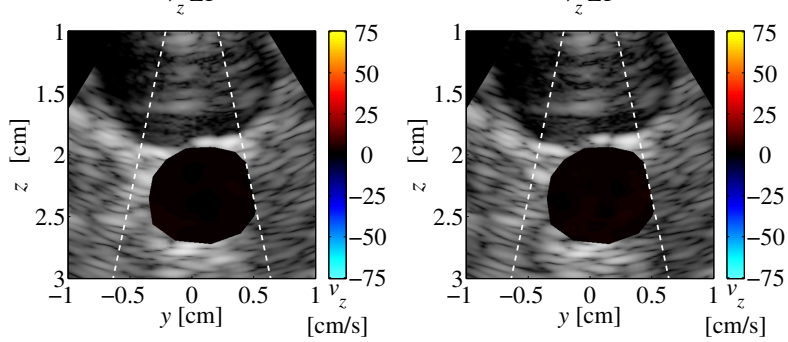

Fig. 2. Magnitude of each velocity component in the ZY-plane for two consecutive frames in the dataset: frame 1 (left) and frame 2 (right). Frame 1 is identified as the end-diastole and frame 2 is identified as peak-systole with maximum out-of-plane $v_{x}$ velocity of $74.3 \mathrm{~cm} / \mathrm{s}$. The two dotted white lines on each image illustrate the span of the flow emissions.

$14.6 \mathrm{~m} / \mathrm{s}$ at end-diastole and $79.3 \mathrm{~m} / \mathrm{s}$ at peak systole (data not shown).

The maximum out-of-plane velocities for the three identified end-diastoles and peak-systoles were $68.5 \pm 5.1 \mathrm{~cm} / \mathrm{s}$ and $26.3 \pm 3.3 \mathrm{~cm} / \mathrm{s}$, respectively. For the longitudinal $\mathrm{ZX}$ plane the three identified end-diastoles and peak-systoles were $65.2 \pm 14.0 \mathrm{~cm} / \mathrm{s}$ and $33.6 \pm 4.3 \mathrm{~cm} / \mathrm{s}$, respectively

Since each velocity estimate is averaged from 32 emissions that are acquired in the time it takes to produce one half plane $(32 \times(5+1)$ emissions $)$, the estimated velocities will always have lower maximum velocities and higher minimum velocities compared to what can be obtained on a commercial scanners with spectral Doppler. This is due to a longer acquisition time that results in a velocity estimate that is averaged over a longer time interval than spectral Doppler.

In Fig. 4, velocity profiles of the out-of-plane $v_{x}$ component can be seen for the two centerlines in frames 1 and 2. The profiles illustrate the out-of-plane velocity at the end-diastole (red curve) and during peak-systole (blue curve). The velocity profile at end-diastole exhibits a more plug flow curve than at the peak-systole. For a 3-D vector velocity representation of the peak-systole flow in the ZY-plane see Fig. 5.
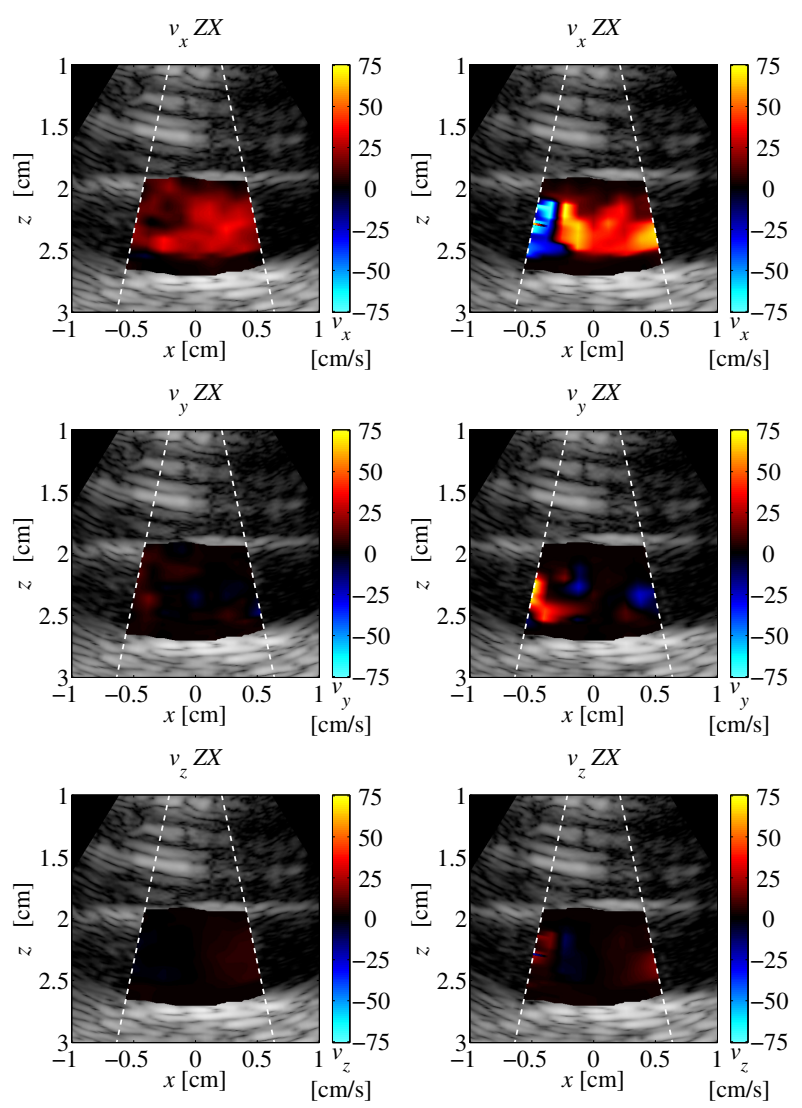

Fig. 3. Magnitude of each velocity component in the ZX-plane for two consecutive frames in the dataset: frame 1 (left) and frame 2 (right). Frame 1 is identified as the end-diastole and frame 2 is identified as peak-systole with maximum $v_{x}$ velocity of $74.9 \mathrm{~cm} / \mathrm{s}$. The two dotted white lines on each image illustrate the span of the flow emissions. The erroneous velocity estimates in the left half plane at the peaksystole might be due to the rapid wall-movement that weakens the effect of the echo canceling filter.

\section{DISCUSSION}

The small active aperture in the 2-D transducer used results in a relatively long lateral wavelength that complicates the estimation of slow flow. Furthermore, the small aperture also complicates the construction of an accurate echo-canceling filter, which is designed to suppress the RF-signal from slowly moving tissue. This is especially seen during peaksystole where the echo-canceling filter has some difficulties to suppress the strong signal from the rapid wall-movement, which can lead to biased velocity estimation. The rapid wallmovement can be an explanation to why erroneous velocity estimates occur during peak-systole in Fig. 3. The velocity estimates in the right half-plane at peak systole in the ZXplane are as expected and are quantitative comparable with the velocities obtained during peak-systole in the ZY-plane (see Fig. 2). Since the emissions sequence obtains data for one halfplane at a time a short time difference is present between the estimates which can explain why erroneous velocity estimates only are present in one plane and that peak and mean velocities differs from the two crossing planes.

Some disadvantages of the sequence used, are that the packet size and the PRF have to be predefined before the measurements, as is normally adjusted in commercial scanners 
$V_{x}$ Velocity profiles in ZY-plane

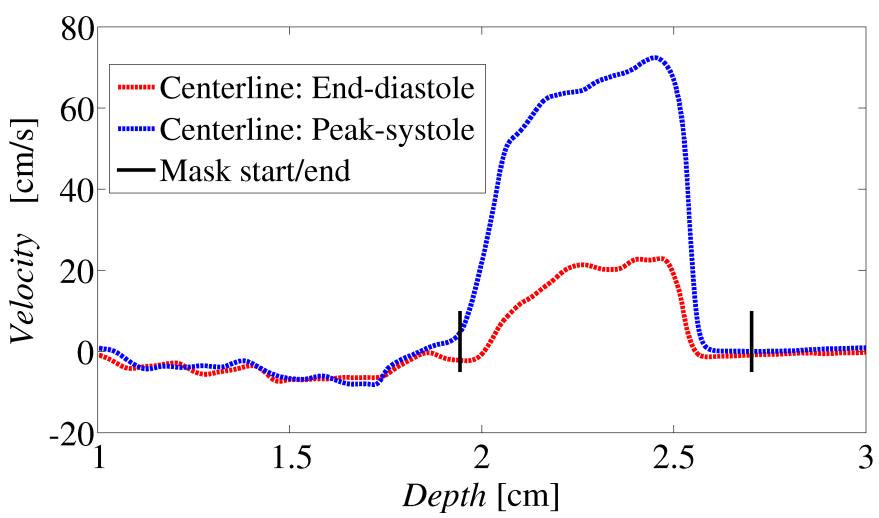

Fig. 4. Velocity profiles for the centerline estimate of the out-of-plane components in the ZY-plane. The red curve shows the $V_{x}$ as a function of depth just before peak systole and the blue curve shows it at the identified peaksystole. The two black lines mark the end and beginning of the discriminator for that line.

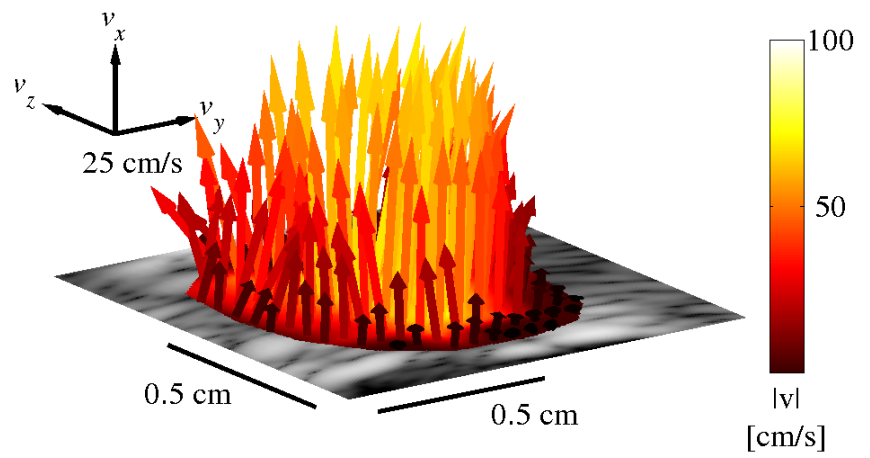

Fig. 5. 3-D vector velocity representation of the blood flow at peak-systole in the ZY-plane.

by the user. For estimating slow flow a low PRF and a large packet size is preferable, whereas for fast flow the opposite is needed for the best velocity estimation.

The TO method can gain from the large transverse wavelength, which makes it possible to estimate large velocities in the lateral and elevation direction. The larger wavelength makes it possible to either lower the PRF or to redesign the sequence and emit more unique flow lines and still be able to estimate all present velocities.

\section{CONCLUSION}

This paper demonstrates for the first time, real-time in vivo 3-D vector velocities measured with US. The obtained $V_{x}$ velocities averaged over three peak-systoles were $15.8 \%$ lower for the ZY-plane and 21.6\% lower for the ZX-plane, than velocities estimated with spectral Doppler using a commercial scanner. Intensity and heat measurements were conducted, both in compliance with the FDA limits. The results show that 3D VFI can be obtained in vivo with a 2-D transducer using the TO method. It is a step towards estimating flow rates for diagnosing cardiovascular diseases without making any assumptions about vessel geometry or angle correction.

\section{ACKNOWLEDGMENT}

This work was supported by grant 82-2012-4 from the Danish Advanced Technology Foundation and by BK Medical Aps.

\section{REFERENCES}

[1] G. M. von Reutern and M. W. Goertler and N. M. Bornstein and M. D. Sette and D. H. Evans and A. Hetzel and M. Kaps and F. Perren and A. Razumovky and et al.", title = "Grading Carotid Stenosis Using Ultrasonic Methods, Stroke, vol. 43, no. 3, pp. 916-921, 2012.

[2] G. R. Hong, M. Kim, G. Pedrizzetti, and M. A. Vannan, "Current clinical application of intracardiac flow analysis using echocardiography," Journal of Cardiovasc Ultrasound, vol. 21, no. 4, pp. 155-162, 2013.

[3] M. Markl, A. Harloff, T. A. Bley, M. Zaitsev, B. Jung, E. Weigang, M. Langer, J. Hennig, and A. Frydrychowicz, "Time-resolved 3D MR velocity mapping at $3 \mathrm{~T}$ : improved navigator-gated assessment of vascular anatomy and blood flow." Journal of magnetic resonance imaging, vol. 25, no. 4, pp. 824-831, 2007.

[4] J. Eriksson, C. Carlhall, P. Dyverfeldt, J. Engvall, A. Bolger, and T. Ebbers, "Semi-automatic quantification of $4 \mathrm{D}$ left ventricular blood flow," Journal of Cardiovascular Magnetic Resonance, vol. 12, no. 1, p. $9,2010$.

[5] M. J. Pihl, M. B. Stuart, B. G. Tomov, P. M. Hansen, M. B. Nielsen, and J. A. Jensen, "In vivo three-dimensional velocity vector imaging and volumetric flow rate measurements," in Proc. IEEE Ultrason. Symp., July 2013, pp. 72-75.

[6] R. W. Gill, "Measurement of blood flow by ultrasound: Accuracy and sources of error," Ultrasound Med. Biol., vol. 11, pp. 625-641, 1985.

[7] J. A. Jensen, H. Holten-Lund, R. T. Nilsson, M. Hansen, U. D. Larsen, R. P. Domsten, B. G. Tomov, M. B. Stuart, S. I. Nikolov, M. J. Pihl, Y. Du, J. H. Rasmussen, and M. F. Rasmussen, "SARUS: A synthetic aperture real-time ultrasound system," IEEE Trans. Ultrason., Ferroelec., Freq. Contr., vol. 60, no. 9, pp. 1838-1852, 2013.

[8] FDA, "Information for manufacturers seeking marketing clearance of diagnostic ultrasound systems and transducers," Center for Devices and Radiological Health, United States Food and Drug Administration, Tech. Rep., 2008.

[9] IEC, "Medical electrical equipment - part 2-37: Particular requirements for the safety of ultrasonic medical diagnostic and monitoring equipment," International Electrotechnical Commision, Tech. Rep., 2004.

[10] J. M. Hansen, M. C. Hemmsen, and J. A. Jensen, "An object-oriented multi-threaded software beamformation toolbox," in Proc. SPIE Med. Imag., vol. 7968, March 2011, pp. 79680 Y 1-9. [Online]. Available: http://dx.doi.org/10.1117/12.878178

[11] M. J. Pihl and J. A. Jensen, "3D velocity estimation using a 2D phased array," in Proc. IEEE Ultrason. Symp., 2011, pp. 430-433.

[12] A. P. G. Hoeks, J. J. W. van de Vorst, A. Dabekaussen, P. J. Brands, and R. S. Reneman, "An efficient algorithm to remove low frequency Doppler signal in digital Doppler systems," Ultrason. Imaging, vol. 13, pp. 135-145, 1991.

[13] C. Kasai, K. Namekawa, A. Koyano, and R. Omoto, "Real-Time TwoDimensional Blood Flow Imaging using an Autocorrelation Technique," IEEE Trans. Son. Ultrason., vol. 32, pp. 458-463, 1985.

[14] J. A. Jensen and P. Munk, "A New Method for Estimation of Velocity Vectors," IEEE Trans. Ultrason., Ferroelec., Freq. Contr., vol. 45, pp. 837-851, 1998.

[15] J. A. Jensen, "A New Estimator for Vector Velocity Estimation," IEEE Trans. Ultrason., Ferroelec., Freq. Contr., vol. 48, no. 4, pp. 886-894, 2001. 